\title{
Caracterización de las complicaciones renales en pacientes con anemia de células falciformes
}

\section{Characterization of kidney complications in patients with sickle cell anemia}

\author{
Isaza-López MC. ${ }^{\mathrm{a}, \mathrm{b}}$, Rojas-Rosas LF. ${ }^{\mathrm{a}, \mathrm{c}}$, Echavarría-Ospina L. ${ }^{\mathrm{d}}$, Serna-Higuita LM. ${ }^{\mathrm{e}}$ \\ aDepartamento de Pediatría. Hospital General de Medellín, Colombia \\ bEscuela Ciencias de la Salud. Universidad Pontificia Bolivariana, Medellín, Colombia \\ 'Escuela Ciencias de la Salud. Corporación Universitaria Remington, Medellín, Colombia \\ dDepartamento de Gastroenterología, Clínica Medellín, Colombia \\ eUniversidad de Tübingen, Alemania
}

Recibido: 11 de junio de 2019; Aceptado: 01 de octubre de 2019

\section{¿Qué se sabe del tema que trata este estudio?}

La nefropatía falciforme es una complicación frecuente de la enfermedad por anemia de células falciformes que comprende alteraciones renales tubulares y glomerulares, que tradicionalmente se manifiestan en la edad adulta con microalbuminuria y glomeruloesclerosis.

\begin{abstract}
¿Qué aporta este estudio a lo ya conocido?
Encontramos hallazgos de nefropatía falciforme desde la etapa de lactante, principalmente hiperfiltración glomerular, microalbuminuria, lesión renal aguda, hipertensión arterial e hipostenuria. La presencia de hipertensión pulmonar o dilatación de cavidades cardíacas, se documentó más frecuentemente en pacientes con nefropatía falciforme
\end{abstract}

\section{Resumen}

La nefropatía falciforme (NF) es una complicación poco estudiada en la edad pediátrica, que se manifiesta en diferentes formas, incluyendo la glomerulopatía y la tubulopatía. Objetivo: Describir las complicaciones renales agudas y crónicas de niños con anemia de células falciformes (ACF). Pacientes y Método: Estudio de cohorte restrospectiva. Se incluyeron pacientes pediátricos con diagnóstico confirmado de enfermedad de células falciformes que tuvieran estudio nefro-urológico. Se consignó patrón electroforético de hemoglobina, presencia y tipo de afectación renal, y presencia de compromiso cardiológico. Se realizó análisis bivariado para comparar pacientes con y sin NF. Resultados: Se incluyeron 79 pacientes, $59.5 \%$ hombres, siendo el patrón electroforético más frecuente Hb-SS (60.9\%). La NF se presentó en el 70\% de ellos, con una edad de 114 meses (RIQ 65-157). Las alteraciones más frecuentemente encontradas fueron hiperfiltración glomerular, microalbuminuria, lesión renal aguda, hipertensión arterial e hipostenuria. En el análisis bivariado, un ecocardiograma anormal fue más frecuente en los pacientes con NF $(84,8 \%$ vs $54,3 \% \mathrm{p}=0,01)$, así como tuvieron una tendencia a mayor uso de medicamentos nefrotóxicos $(74,5 \%$ vs $54,2 \% \mathrm{p}=0,07)$. Conclusiones: Nuestros hallazgos sugieren que la nefropatía falciforme puede presentarse a temprana edad, siendo muy frecuente la hiperfiltración glomerular. Las complicaciones cardiopulmonares en ACF se podrían asociar con la presencia NF.
Palabras clave: Anemia de Células Falciformes; Proteinuria; Insuficiencia renal; Nefropatía falciforme

Correspondencia:

Isaza-López MC.

mariac.isaza@upb.edu.co

Como citar este artículo: Rev Chil Pediatr. 2020;91(1):51-57. DOI: 10.32641/rchped.v91i1.1274 


\begin{abstract}
Sickle cell nephropathy (SCN) is a poorly studied complication of pediatric patients. It appears in different forms, including glomerulopathy, and tubulopathies. Objective: To describe acute and chronic renal complications in patients with sickle cell anemia (SCA). Patients and Method: Retrospective study. Pediatric patients with confirmed diagnosis of sickle cell disease were included who had a nephro-urology study. Hemoglobin electrophoresis pattern, presence and type of renal involvement, and presence of cardiac involvement were recorded. Bivariate analysis was performed to compare patients with and without SCN. Results: 79 patients were included, 59.5\% of them were men, and the most frequent electrophoresis pattern was Hb-SS (60.9\%). The SCN occurred in $70 \%$ of patients with an average age of 114 months (RIQ 65-157). The most frequently observed alterations were glomerular hyperfiltration, microalbuminuria, acute kidney injury, arterial hypertension, and hyposthenuria. In the bivariate analysis, an abnormal echocardiogram result was presented more frequently in patients with $\operatorname{SCN}(84.8 \%$ vs. $54.3 \% \mathrm{p}=0.01)$, as well as more frequent use of nephrotoxic drugs $(74.5 \%$ vs. $54.2 \% \mathrm{p}=0.07)$. Conclusions: Our findings suggest that sickle cell nephropathy may occur at an early age, where glomerular hyperfiltration is very common. Cardiopulmonary complications in patients with SCA may be related to the presence of SCN.
\end{abstract}

Keywords:

Sickle Cell Anemia;

Proteinuria;

Renal failure;

sickle cell nephropathy

\section{Introducción}

La anemia de células falciformes (ACF) es una enfermedad hematológica autosómica recesiva de alta prevalencia ${ }^{1}$. La ACF se produce por mutaciones en el cromosoma 11 (11p15.4) que causan una alteración estructural en la subunidad $\beta$ de la hemoglobina ${ }^{1,2}$ y llevan a la producción de la Hemoglobina S $(\mathrm{Hb} \mathrm{S})^{1}$. Esta hemoglobina se caracteriza por formar polímeros de $\mathrm{Hb}$ ante la presencia de hipoxia tisular, hiperosmolaridad y acidosis; produciendo cambios en la membrana y deformando los glóbulos rojos ${ }^{3}$. Los eritrocitos con hemoglobina polimerizada son rígidos y en forma de media luna ${ }^{2}$, lo cual induce hemólisis y fenómenos de vasooclusión recurrentes en los vasos de pequeño calibre, generando isquemia e inflamación tisular; provocando crisis dolorosas, aumento en la susceptibilidad de infecciones e incluso daño de órgano.

Entre las complicaciones secundarias a la ACF se encuentran las complicaciones renales, conocidas como la nefropatía falciforme $(\mathrm{NF})^{2,3}$. Las NF comprende tanto alteraciones glomerulares como tubulares, entre ellas la hematuria, hipostenuria, desórdenes tubulares e insuficiencia renal tanto aguda como crónica; hallazgos que pueden manifestarse tempranamente en la edad pediátrica ${ }^{2-4}$, aumentando la morbimortali$\mathrm{dad}^{5}$. Desafortunadamente existen pocos estudios que evaluen la presencia de NF a temprana edad y qué factores de riesgo se asocian a su desarrollo ${ }^{2}$. Este estudio tiene como objetivo describir prevalencia de NF, factores de riesgo y su caracterización, en una población pediátrica con ACF.

\section{Pacientes y Método}

\section{Recolección de la información}

Estudio tipo cohorte retrospectiva donde se revisó la historia clínica electrónica de los pacientes pediátricos atendidos en el Hospital General de Medellín durante el período 2009-2017. Se incluyeron pacientes con diagnóstico confirmado de enfermedad de células falcifomes con y sin crisis falciforme que tuvieran algún estudio nefro-urológico.

Se evaluaron las características sociodemográficas y clínicas de los pacientes, se registró el resultado de la electroforesis de hemoglobina, se buscó la presencia de hipertensión arterial (HTA) según las recomendaciones de la Academia Americana de Pediatría del año $2017^{6}$ y se verificó la presencia de hipertensión pulmonar (HP) mediante los hallazgos ecocardiográficos reportados en el último año de seguimiento; se consideró HP cuando la velocidad pico de flujo de insuficiencia tricuspídea fue mayor o igual a $2,5 \mathrm{~m} / \mathrm{seg}$ que se corresponde indirectamente con una presión de arteria pulmonar mayor a $35 \mathrm{mmHg}^{7}$. Se evaluaron además los tratamientos farmacológicos recibidos y el requerimiento de hemoderivados.

Para el diagnóstico de NF, se utilizó las diferentes definiciones reportadas en la literatura ${ }^{3,8}$, donde se considera NF la afectación renal secundaria a la ACF y comprende un amplio espectro de manifestaciones que incluyen cualquiera de los siguientes hallazgos: hipostenuria (gravedad específica menor de 1.010), proteinuria (mayor o igual a $30 \mathrm{mg} / \mathrm{dL}$ ), hematuria (mayor o igual a tres eritrocitos por campo de alto poder de una gota de orina sin centrifugar), microalbuminuria 
(excreción de albúmina en orina ocasional mayor a 30 $\mathrm{mg} / \mathrm{g}$ ), alteración en el doppler de vasos renales, necrosis papilar y alteración de la tasa de filtración glomerular (TFG) $)^{2,8}$. La TFG se calculó con la fórmula de Schwartz $($ TFG $=$ Talla $(\mathrm{cm}) \times 0,413 /$ creatinina plasmática $(\mathrm{mg} / \mathrm{dL})$. En los pacientes con deterioro de la función renal se utilizó la clasificación de $\mathrm{KDIGO}^{9}$ para estadificar la lesión renal aguda (LRA). Se consideró hiperfiltración glomerular un aumento de la TFG por encima 2 DE de lo normal para la edad así: niño entre 6 meses- 1 año de $117,2 \mathrm{ml} / \mathrm{min} / 1,73 \mathrm{~m}^{2}$, entre $1-2$ años $130,7 \mathrm{ml} / \mathrm{min} / 1,73 \mathrm{~m}^{2}$ y mayores de 2 años un valor de $145,2 \mathrm{ml} / \mathrm{min} / 1,73 \mathrm{~m}^{2}{ }^{10}$.

\section{Análisis estadístico}

Se utilizó estadística descriptiva, las variables cualitativas se analizaron como proporciones y valores absolutos; las variables cuantitativas se describieron con medias y desviación estandar o medianas y rangos intercuartílicos (RIQ) según la distribución de los datos, la normalidad se evaluó por medio de histogramas, Q-Q plots, Box-plot, kurtosis y asimetría. Posteriormente se hizó una análisis bivariado comparando los pacientes con NF contra los paciente sin presencia de NF, se utilizó la prueba de Chi cuadrado o Fisher de acuerdo al número de datos en las casillas. Un valor de $\mathrm{p}<0,05$ fue considerado estadísticamente significativo. El análisis estadístico fue realizado con el programa SPSS versión 25 .

\section{Consideraciones éticas}

Este estudio fue aprobado por el comité de investigaciones del Hospital General de Medellín, y se siguieron las normas sobre aspectos éticos de la investigación en seres humanos contenidas en la resolución 00843 de 1993, emitida por el Ministerio de Salud de Colombia; por otro lado este estudio fue clasificado como una investigación sin riesgo para los pacientes.

\section{Resultados}

Durante 9 años de observación, 119 pacientes con diagnóstico confirmado de enfermedad de células falciformes fueron atendidos en el centro hospitalario; de los cuales 40 pacientes fueron excluidos por no tener ningún estudio renal, quedando un total de 79 pacientes. En 29 pacientes se registró más de un ingreso hospitalario (136 eventos). La mediana de edad fue de 114 meses (RIQ 75-156) y el 59,5\% fueron hombres. De los 79 pacientes incluídos con diagnóstico de enfermedad de células falciforme, se reportó el patrón electroforético en 46 pacientes, de los cuales 28 (60,9\%) correspondió a Hb SS; mientras que 8 de ellos $(17,4 \%)$, fueron rasgo falciforme. En los 33 pacientes restantes con diagnóstico conocido de enfermedad de células falcifomes no se registró el patrón electroforético.

Entre las principales causas de ingreso en esta serie se encontraron las crisis dolorosas $84,8 \%$, crisis hemolíticas $78,5 \%$, infecciones $49,4 \%$ y síndrome torácico agudo 33\% (tabla 1). Del total de pacientes, $75,9 \%$ (RIQ 60-79) recibió transfusión de glóbulos rojos durante el evento agudo y solo seis pacientes tenían protocolo de hipertransfusión crónica. Entre las complicaciones observadas, $12.7 \%$ de los pacientes presentaron HTA, y $34 \%$ alguna alteración electrolítica.

\begin{tabular}{|c|c|c|}
\hline Variable & $\mathrm{n}$ & \\
\hline Sexo hombre n (\%) & 79 & $47(59,5 \%)$ \\
\hline Peso mediana (RIQ) & 79 & $25,5 \mathrm{~kg}(18-31)$ \\
\hline Talla media $( \pm D E)$ & 79 & $126,4 \mathrm{~cm}( \pm 21,1)$ \\
\hline Edad al diagnóstico de ACF mediana (RIQ) & 79 & 15,5 meses $(8-48)$ \\
\hline Edad mediana de NF (RIQ) & 55 & 114 meses $(75-156)$ \\
\hline $\begin{array}{l}\text { Patrón electrofóretico n (\%) } \\
\text { Hb SS } \\
\text { Hb SC } \\
\text { Hb SB+ } \\
\text { Hb SD } \\
\text { Rasgo falciforme }\end{array}$ & 46 & $\begin{array}{r}28(60,9 \%) \\
5(10,9 \%) \\
4 \quad(8,7 \%) \\
1 \quad(2,2 \%) \\
8(17,4 \%)\end{array}$ \\
\hline Transfusión aguda glóbulos rojos n (\%) & 79 & $60(75,9 \%)$ \\
\hline Hipertransfusiones crónicas n (\%) & 79 & $6(7,7 \%)$ \\
\hline Crisis dolorosa n (\%) & 79 & $67(84,8 \%)$ \\
\hline Crisis hemolítica n (\%) & 79 & $62(78,5 \%)$ \\
\hline Sepsis n (\%) & 79 & $39(49,4 \%)$ \\
\hline Síndrome torácico agudo n (\%) & 79 & $26(32,9 \%)$ \\
\hline ECV n (\%) & 79 & $6(7,6 \%)$ \\
\hline Secuestro esplénico n (\%) & 79 & $3(3,8 \%)$ \\
\hline Otras complicaciones n (\%) & 79 & $25(31,6 \%)$ \\
\hline $\begin{array}{l}\text { Ecocardiograma n (\%) } \\
\text { Normal } \\
\text { Anormal }\end{array}$ & 61 & $\begin{array}{l}14(23 \%) \\
47(59,5 \%)\end{array}$ \\
\hline Hipertensión arterial n (\%) & 79 & $10(12,7 \%)$ \\
\hline Hemoglobina media $( \pm \mathrm{DE})$ & 79 & $7,5 \mathrm{mg} / \mathrm{dL}( \pm 1,97)$ \\
\hline Creatinina media $( \pm \mathrm{DE})$ & 79 & $0,37 \mathrm{mg} / \mathrm{dL}( \pm 0,10)$ \\
\hline BUN median (RIQ) & 79 & $8(5,9-10,0)$ \\
\hline $\begin{array}{l}\text { TFG media } \\
( \pm \mathrm{DE})\end{array}$ & 79 & $\begin{array}{l}152,4 \mathrm{~mL} / 1,73 \mathrm{~m}^{2} / \mathrm{min} \\
( \pm 36,9)\end{array}$ \\
\hline Terapia de remplazo renal (\%) & 79 & $0(0 \%)$ \\
\hline Mortalidad n (\%) & 79 & $0(0 \%)$ \\
\hline
\end{tabular}

ACF: anemia de células falciformes; NF: nefropatía falciforme; ECV: enfermedad cerebro vascular; TFG: tasa de filtración glomerular; $\mathrm{Hb}$ : hemoglobina; BUN: nitrogeno ureico. 
En 62 pacientes se realizó un ecocardiograma, de los cuales el 59,5\% (RIQ 47-61) de los pacientes presentaron alguna anormalidad en el ecocardiograma (HP y/o dilatación de cavidades ventriculares). Con respecto a la terapia suministrada para el tratamiento crónico ambulatorio de la ACF, el 49,4\% de los pacientes recibía hidroxiurea.

La NF se presentó en el 70\%; la edad de detección de la primera complicación renal fue de 114 meses (RIQ 65-157). Las alteraciones renales encontradas fueron hipostenuria en el 11,4\%, hematuria 3,8\%, pH urinario anormal 5,1\%. En 21 pacientes se evaluó la presencia de microalbuminuria, encontrándose en el 19\% (figura 1). La media de la TFG fue de $152 \pm 36,9$ $\mathrm{mL} / \mathrm{min} / 1,73 \mathrm{~m}^{2}$. La presencia de hiperfiltración glomerular fue documentada en el 59,3\% de los pacientes en quienes se estimó la TFG. En 73 pacientes se realizó medición de creatinina sérica, con una media de $0,37 \pm 0,1 \mathrm{mg} / \mathrm{dl}$. Se encontró LRA en 10 pacientes, 4 clasificados como KDIGO 1, 2 como KDIGO 2 y 4 pacientes KDIGO 3. Una paciente tuvo dos episodios de LRA durante el período de observación. Del total de pacientes con presencia de LRA; los valores de creatinina sérica se normalizaron en el 90\% de los casos y en un paciente no se tomó control de función renal.

Las complicaciones de la ACF asociados a la presentación de LRA fueron crisis hemolítica con requerimiento de transfusión de glóbulos rojos en todos los pacientes, crisis vaso-oclusiva en $9 / 10$ pacientes $y$ sepsis en 8/10 pacientes. Se documentó HTA en 3/10 pacientes con LRA, dos de ellos debutaron con emer- gencia hipertensiva con órgano blanco cerebro y riñón, respectivamente. En 8/10 pacientes se observaron cambios ecocardiográficos en el contexto de ACF (HP y/o dilatación de cavidades ventriculares).

En la tabla 2 se presentan las complicaciones agrupadas según el comportamiento clínico de la de enfermedad de células falciformes (71 pacientes con anemia de falciforme contra 8 pacientes de rasgo falciforme). Se puede observar que la presencia de hiperfiltración fue mas frecuente en los pacientes con ACF en comparación con los pacientes con rasgo (62,1\% vs $28,6 \%)$; adicionalmente el porcentaje de pacientes con sedimento urinario alterado fue menor en los pacientes con ACF en comparación con los pacientes con rasgo falciforme $(4,2 \%$ vs $37,5 \%)$. Sin embargo, por el pequeño número de pacientes en el grupo rasgo falciforme, no es posible determinar si esta diferencia es estadísticamente significativa.

En el análisis bivariado, al comparar los pacientes con NF con aquellos sin nefropatía, no se encontró diferencias con respecto a la distribución por sexo, patrón electroforético, transfusión de glóbulos rojos, hipertransfusión crónica y uso de hidroxiurea (tabla 3). Por el contrario, un resultado anormal del ecocardiograma se presentó en forma mas frecuente en los pacientes con $\mathrm{NF}$ ( 83 vs 53,3\%, $\mathrm{p}=0,02$ ). Adicionalmente se observó una tendencia a mayor uso de medicamentos nefrotóxicos en los pacientes con diagnóstico de NF (74,5\% vs 54,2\%, p = 0,07). Durante el período de seguimiento ningún paciente presentó enfermedad renal crónica o necesidad de terapia de reemplazo renal; tampoco se reportó ninguna muerte.
Figura 1. Complicaciones renales observadas en los pacientes con enfermedad de células falciformes. LRA: lesión renal aguda; Sed: sedimento; $\mathrm{pHU}$ : $\mathrm{pH}$ en orina.

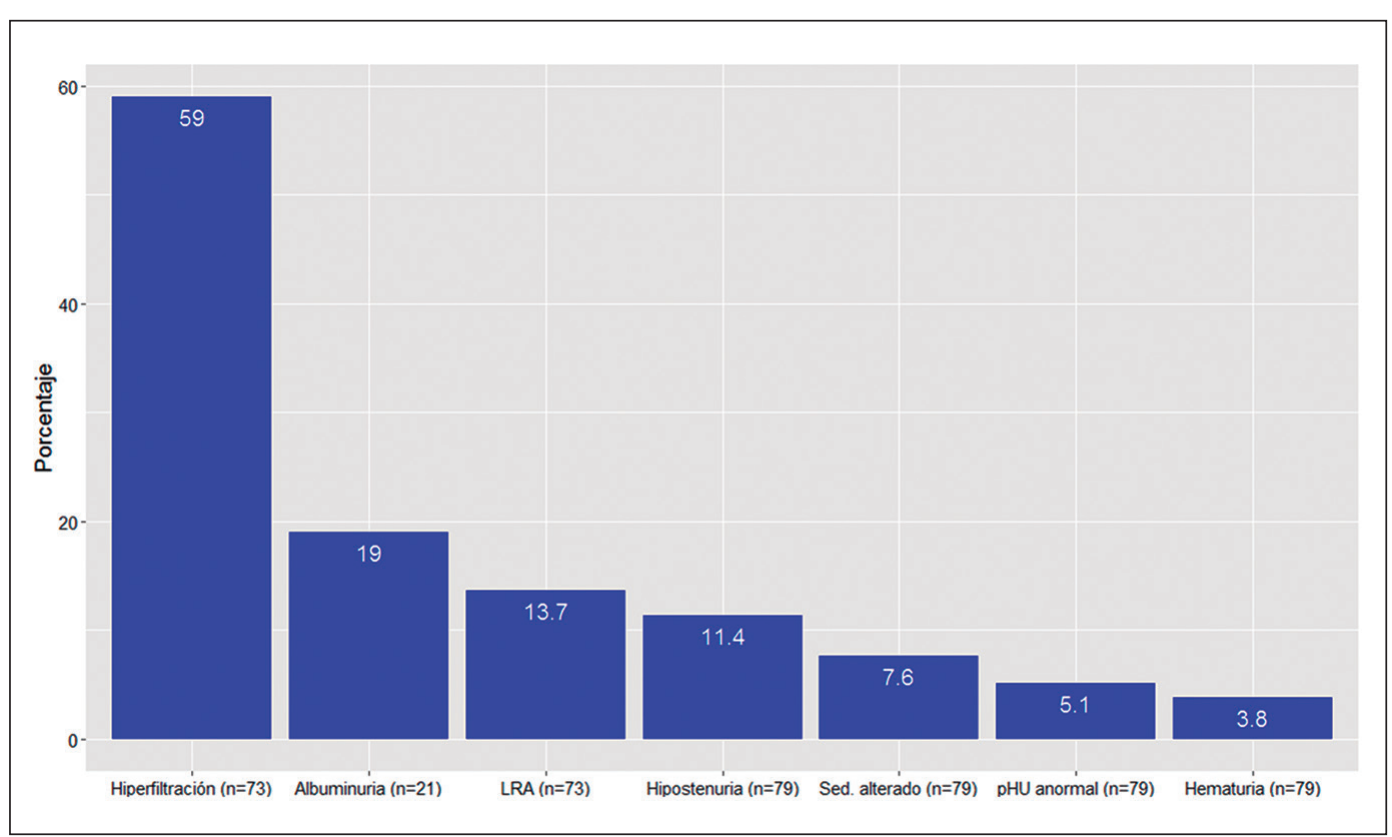


Tabla 2. Complicaciones observadas en los pacientes con enfermedad de células falciformes agrupadas según el perfil clínico de la enfermedad

\begin{tabular}{llcl}
\hline & & Anemia de células falciformes $(n=71)$ & Rasgo falciforme $(n=8)$ \\
\hline Nefropatía falciforme & $(n=79)$ & $49(69 \%)$ & $6(75 \%)$ \\
Hiperfiltración glomerular & $(n=73)$ & $41(62,1 \%)$ & $2(28,6 \%)$ \\
Albuminuria & $(n=21)$ & $4(2,2 \%)$ & $0(0 \%)$ \\
LRA & $(n=73)$ & $9(13,8 \%)$ & $1(12,5 \%)$ \\
Hipostenuria & $(n=79)$ & $9(12,7 \%)$ & $0(0 \%)$ \\
Sedimento alterado & $(n=79)$ & $3(4,2 \%)$ & $3(37,5 \%)$ \\
pH Urinario anormal & $(n=79)$ & $4(5,6 \%)$ & $0(0 \%)$ \\
Hematuria & $(n=79)$ & $3(4,2 \%)$ & $0(0 \%)$ \\
HTA & $(n=79)$ & $8(11,3 \%)$ & $2(25 \%)$ \\
Ecocardiograma anormal & $(n=62)$ & $42(75 \%)$ & $5(83,3 \%)$ \\
\hline
\end{tabular}

LRA: Lesión renal aguda; Sed: sedimento; HTA: hipertensión arterial.

Tabla 3. Análisis bivariado, según la presencia o no de nefropatía falciforme

\begin{tabular}{lcccc}
\hline & & Nefropatía falciforme $(\mathrm{n}=55)$ & No nefropatía falciforme $(\mathrm{n}=24)$ & Valor de $\mathrm{p}$ \\
\hline Sexo masculino & $\mathrm{n}(\%)$ & $32(58,2 \%)$ & $15(62,5 \%)$ & 0,72 \\
Hb SS $(\mathrm{n}=46)$ & $\mathrm{n}(\%)$ & $21(63,6 \%)$ & $7(53,8 \%)$ & 0,76 \\
Tranfusión aguda glóbulos rojos & $\mathrm{n}(\%)$ & $44(80 \%)$ & $16(66,7 \%)$ & 0,20 \\
Hipertransfusiones crónicas & $\mathrm{n}(\%)$ & $5(9,3 \%)$ & $1(4,2 \%)$ & 0,44 \\
Ecocardiograma anormal $(\mathrm{n}=62)$ & $\mathrm{n}(\%)$ & $39(83,0 \%)$ & $8(53,3 \%)$ & 0,02 \\
Uso crónico de hidroxiúrea & $\mathrm{n}(\%)$ & $29(52,7 \%)$ & $10(41,7 \%)$ & 0,37 \\
Exposición nefrotóxicos & $\mathrm{n}(\%)$ & $41(74,5 \%)$ & $13(54,2 \%)$ & 0,07 \\
\hline
\end{tabular}

$\mathrm{Hb}$ : hemoglobina.

\section{Discusión}

En el presente estudio se evaluó retrospectivamente una cohorte de 79 pacientes con diagnóstico de ACF, encontrando una prevalencia de NF del 70\%; definida como la afectación renal secundaria a la ACF que se manifiesta con alteraciones tanto tubulares como glomerulares ${ }^{2,3,8}$. Las alteraciones renales más frecuentes encontradas fueron hiperfiltración glomerular, microalbuminuria, HTA y LRA.

Las recomendaciones actuales sugieren la realización del cribado renal a partir de los 10 años de edad ${ }^{11}$, lo cual contribuye a la baja detección de la NF durante la infancia. Sin embargo, otros autores sugieren iniciar el cribado a una edad más temprana ${ }^{12,13}$. En nuestra serie la edad media de detección de la primera complicación renal fue 114 meses (RIQ 65-157), similar a lo reportado en estudios previos donde la edad de detección fue entre los 7 a 9 años de edad ${ }^{12,14}$; sin embargo, hay reportes de complicaciones renales de aparición temprana como hiperfiltración glomerular diagnosticada a los 13 meses $^{15}$ o microalbuminuria a los 2,8 años ${ }^{5,12}$

En relación al patron electroforético, en 33 pacientes con diagnóstico previo de ACF y perfil clínico compatible con ACF (crisis vaso-oclusivas y hemolíticas frecuentes, alto requerimiento transfusional), no se registró el resultado del patrón electroforético; lo cual constituye una limitante de este estudio. El tamaño pequeño de los grupos de pacientes con ACF y rasgo falciforme, no permite afirmar que existe una diferencia en cuanto al desenlace de nefropatía falciforme. Recientemente una revisión sistématica de pacientes con rasgo falciforme, no reportó asociación con complicaciones renales en los estudios pediátricos incluídos; más si, una asociación positiva para proteinuria y enfermedad renal crónica en adultos con rasgo falciforme ${ }^{16}$.

Adicionalmente, algunos estudios reportan una mayor incidencia de alguna manifestación de NF o 
edad de presentación más temprana de la misma en los pacientes con un patrón electroforético $\mathrm{Hb} \mathrm{SS}^{12,14}$. Estos pacientes presentan un curso más agresivo de la enfermedad falciforme por el daño de órgano blanco secundario a las crisis vaso-oclusivas frecuentes ${ }^{8}$. Un estudio en República del Congo encontró una prevalencia de hiperfiltración glomerular del 30.8\% en niños con $\mathrm{Hb}$ SS comparado con 6,1\% del grupo con $\mathrm{Hb}$ $\mathrm{AA}^{17}$. En nuestra serie, el $63,6 \%$ de los pacientes con NF tenían patrón electroforético Hb SS.

La hiperfiltración glomerular se ha descrito como un marcador temprano de afectación renal, la cual contribuye al daño glomerular y al desarrollo de enfermedad renal. Entre los mecanismos fisiopatológicos se ha propuesto que la anemia crónica causa inicialmente vasodilatación renal con la consecuente hiperperfusión e hiperfiltración glomerular ${ }^{8}$, además de la polimerización de la $\mathrm{Hb} \mathrm{S}$ en la médula renal ${ }^{18}$. Nosotros encontramos que el 59,3\% de los pacientes presentaron hiperfiltración glomerular, similar a lo reportado por King y colaboradores $(50,8 \%)^{12}$. En un estudio para cribado de enfermedad renal en 206 niños con ACF se encontró hiperfiltración glomerular en el 84,95\% y una prevalencia de $\mathrm{Hb}$ SS de $92,5 \%{ }^{14}$. En la literatura se ha descrito que la fórmula de Schwartz puede sobrestimar la TFG y en consecuencia aumentar la prevalencia de hiperfiltración glomerular ${ }^{2}$; sin embargo, estudios realizados en lactantes con ACF donde se calculó la TFG mediante renograma con DTPA, también reportaron hiperfiltración glomerular ${ }^{4,15,19}$.

Con respecto a otros factores de riesgo para NF, nosotros encontramos que el $74,5 \%$ de los pacientes con NF recibió algún medicamento nefrotóxico. Hallazgo similar a lo reportado en una investigación de 305 niños con ACF donde el uso de AINES se asoció a microalbuminuria, (ibuprofeno OR 2,3 IC 95\% 1,4-3,8 $\mathrm{p}=0,001$, diclofenaco OR 2 IC 95\% 1,2-3,4 $\mathrm{p}=0,01)^{20}$. Otro estudio realizado en 197 pacientes pediátricos con ACF reportan un mayor riesgo de LRA en los pacientes que recibieron ketorolaco (OR 1,63 IC $95 \% 1,08-2,47)^{21}$. Este hallazgo se explica debido a que los AINES inhiben la acción de las prostaglandinas; lo cual bloquea la respuesta vasodilatadora compensatoria contra la isquemia y vasoconstricción llevando a una mayor disminución del flujo sanguíneo renal durante las crisis vaso-oclusivas ${ }^{21-23}$.

En la literatura se ha descrito una incidencia de 5-18\% de LRA la cual es multifactorial y autolimitada, pero está asociada con mayor mortalidad, falla orgánica múltiple y mayor estancia hospitalaria ${ }^{21,22,24}$. Recientemente se evalúo la presencia de LRA en pacientes pediátricos con ACF y crisis vasooclusiva ${ }^{21}$; encontrándose LRA en el 17\% de ellos. En nuestra investigación, la prevalencia de LRA fue de $13,7 \%$, y todos los eventos ocurrieron durante una crisis hemo- lítica. Entre las posibles explicaciones a esta asociación se encuentra la depleción de volumen, presente en los pacientes con crisis hemolítica, lo cual puede llevar a una LRA ${ }^{22}$.

La prevalencia de HP en niños con ACF es de $24,4 \%{ }^{25}$ y está asociada con mayor frecuencia de crisis hemolíticas, hipoxemia, síndrome torácico agudo ${ }^{26}$, evento cerebrovascular agudo y mayor mortalidad ${ }^{27}$. En nuestro estudio, la presencia de HP y/o dilatación de cavidades ventriculares fue observado más frecuentemente en los pacientes con NF. La coexistencia de complicaciones cardiovasculares y NF a causa de los cambios hemodinámicos producidos por hemólisis, anemia, hipoxemia crónica y posteriormente vasculopatía, es una interesante asociación descrita en adul$\operatorname{tos}^{27,28}$, que no ha sido reportada en pediatría previamente. Futuros estudios debieran dilucidar las razones de esta asociación.

\section{Limitaciones}

Por tratarse de un estudio retrospectivo, la exclusión de aquellos pacientes sin estudios nefro-urológicos completos puede subestimar o sobreestimar la presencia de NF. Así también, la no disponibilidad de la electroforesis de hemoglobina en la totalidad de la cohorte evaluada limita el estudio de su rol. También existen limitaciones derivadas de la realización de un estudio de un solo centro. Consideramos se requieren estudios prospectivos multicéntricos para evaluar con mayor profundidad la NF en la población pediátrica.

\section{Conclusiones}

Los datos disponibles en esta cohorte de pacientes pediátricos con diagnóstico de ACF, sugieren que la NF puede presentarse en forma frecuente antes de los 10 anos de edad; al igual que han sugerido otros autores, nuestra recomendación es iniciar la tamización para NF a una edad mas temprana, especialmente en los pacientes con patrón electroforético $\mathrm{Hb}$ SS, crisis falciforme frecuentes, o ante la presencia de anormalidades cardiopulmonares tipo HP y dilatación de las cavidades ventriculares, las cuales podrían asociarse con la presencia de NF.

\section{Responsabilidades Éticas}

Protección de personas y animales: Los autores declaran que los procedimientos seguidos se conformaron a las normas éticas del comité de experimentación humana responsable y de acuerdo con la Asociación Médica Mundial y la Declaración de Helsinki. 
Confidencialidad de los datos: Los autores declaran que han seguido los protocolos de su centro de trabajo sobre la publicación de datos de pacientes.

Derecho a la privacidad y consentimiento informado: Los autores han obtenido el consentimiento informado de los pacientes y/o sujetos referidos en el artículo. Este documento obra en poder del autor de correspondencia.

\section{Conflicto de intereses}

Los autores declaran no tener conflicto de intereses.

\section{Referencias}

1. Zúñiga CP, Martínez CG, González LMR, et al. Enfermedad de células falciformes: Un diagnóstico para tener presente. Rev. Chil. Pediatría 2018; 0-0. doi:10.4067/ S0370-41062018005000604.

2. Olaniran KO, Eneanya ND, Nigwekar SU, et al. Sickle Cell Nephropathy in the Pediatric Population. Blood Purif. 2019;47:205-13.

3. Aeddula NR, Baradhi KM. Sickle Cell Nephropathy. in StatPearls (StatPearls Publishing, 2019).

4. Aygun B, Mortier NA, Smeltzer MP, Hankins JS, Ware RE. Glomerular hyperfiltration and albuminuria in children with sickle cell anemia. Pediatr. Nephrol. 2011;26:1285-90.

5. Aloni MN, Mabidi JL, Ngiyulu RM, et al. Prevalence and determinants of microalbuminuria in children suffering from sickle cell anemia in steady state. Clin. Kidney J. 2017;10:479-86.

6. Flynn JT, Kaelber DC, BakerSmith CM, et al; Subcommittee on Screening and Management of High Blood Pressure in Children. Clinical Practice Guideline for Screening and Management of High Blood Pressure in Children and Adolescents. Pediatrics. 2017;140(3):e20171904. Pediatrics 142, (2018).

7. Gladwin MT, Vichinsky E. Pulmonary Complications of Sickle Cell Disease. N Engl J Med. 2008;359:2254-65.

8. Nath KA, Hebbel RP. Sickle cell disease: renal manifestations and mechanisms. Nat Rev Nephrol. 2015;11:161-171.

9. Sutherland SM, Byrnes JJ, Kothari M, et al. AKI in Hospitalized Children: Comparing the pRIFLE, AKIN, and KDIGO Definitions. Clin J Am Soc Nephrol. 2015;10:554-61.

10. Pediatric Nephrology. (Springer Berlin
Heidelberg, 2009). doi:10.1007/978-3540-76341-3.

11. Evidence-Based Management of Sickle Cell Disease: Expert Panel Report, 2014. Pediatrics 2014;134:e1775.

12. King L, MooSang M, Miller M, Reid M. Prevalence and predictors of microalbuminuria in Jamaican children with sickle cell disease. Arch Dis Child. 2011;96:1135-9.

13. Wigfall DR, Ware RE, Burchinal MR, Kinney TR, Foreman JW. Prevalence and clinical correlates of glomerulopathy in children with sickle cell disease. J Pediatr. 2000;136:749-53.

14. Garba M, Moussa Tondi ZM, Diongoule $\mathrm{H}$, et al. Dépistage de la néphropathie dans les syndromes drépanocytaires majeurs chez les patients suivis au Centre national de référence de la drépanocytose de Niamey, Niger. Néphrologie Thérapeutique 2018;14:462-466.

15. Ware RE, Rees RC, Sarnaik SA, et al. Renal Function in Infants with Sickle Cell Anemia: Baseline Data from the BABY HUG Trial. J. Pediatr. 2010;156:66-70.e1.

16. Naik RP, Smith-Whitley K, Hassell KL, et al. Clinical Outcomes Associated With Sickle Cell Trait: A Systematic Review. Ann Intern Med. 2018;169(9):619-27.

17. Aloni MN, Ngiyulu RM, Gini-Ehungu $J \mathrm{~L}$, et al. Renal Function in Children Suffering from Sickle Cell Disease: Challenge of Early Detection in Highly Resource-Scarce Settings. PLoS One. 2014;9(5):e96561.

18. de Paula RP, Nascimento AF, Sousa SM, Bastos PR, Barbosa AA. Glomerular filtration rate is altered in children with sickle cell disease: a comparison between $\mathrm{Hb}$ SS and Hb SC. Rev Bras Hematol Hemoter. 2013;35(5):349-51.

19. Aygun B, Mortier NA, Smeltzer MP, Shulkin BL, Hankins JS, Ware RE. Hydroxyurea treatment decreases glomerular hyperfiltration in children with sickle cell anemia. Am J Hematol. 2013;88(2):116-9.

20. Mawanda M, Ssenkusu JM, Odiit A, Kiguli S, Muyingo A, Ndugwa C. Micro-albuminuria in Ugandan children with sickle cell anaemia: a crosssectional study. Ann Trop Paediatr. 2011;31(2):115-21.

21. Baddam S, Aban I, Hilliard L, Howard T, Askenazi D, Lebensburger JD. Acute kidney injury during a pediatric sickle cell vaso-occlusive pain crisis. Pediatr Nephrol. 2017;32(8):1451-6.

22. López Revuelta K, Ricard Andrés MP. Kidney abnormalities in sickle cell disease. Nefrologia. 2011;31(5):591-601.

23. Mammen C, Bissonnette ML, Matsell DG. Acute kidney injury in children with sickle cell disease-compounding a chronic problem. Pediatr Nephrol. 2017;32(8):1287-91.

24. Rémy P, Audard V, Galactéros F. Rein et hémoglobinopathies. Néphrologie Thérapeutique 2016;12:117-29.

25. Musa BM, Galadanci NA, Coker M, Bussell S, Aliyu MH. The global burden of pulmonary hypertension in sickle cell disease: a systematic review and meta-analysis. Ann Hematol 2016;95:1757-64.

26. Minniti CP, Sable C, Campbell A, et al Elevated tricuspid regurgitant jet velocity in children and adolescents with sickle cell disease: association with hemolysis and hemoglobin oxygen desaturation. Haematologica. 2009 Mar;94(3):340-7.

27. Gladwin M.T. Cardiovascular complications and risk of death in sicklecell disease. Lancet 2016;387:2565-74.

28. Novelli EM, Hildesheim M, Rosano C, et al. Elevated Pulse Pressure is Associated with Hemolysis, Proteinuria and Chronic Kidney Disease in Sickle Cell Disease. PLoS One. 2014;9(12):e114309. 